\section{On the Structure of Minimum Effort Solutions with Application to Kinematic Redundancy Resolution}

\author{
Ian A. Gravagne, Member, IEEE, and Ian D. Walker, Member, IEEE
}

robotics community which depends upon the concept of minimum effort [9], [10], [19], we believe minimum effort techniques will prove an invaluable tool in the future, provided their properties and limitations are well understood.

\section{BACKGROUND}

The most commonly found example of underdetermined systems in the robotics literature is probably that of kinematically redundant manipulators, which have been the subject of extensive research over the last few years (see, for example, the survey papers [4], [5]). For these types of manipulators, there are more degrees of freedom (joints) than specified end effector (task) variables, and the key issue is how to exploit this overabundance of joints in the system.

More specifically, given end effector, or task variables, $\underline{x} \in R^{m}$, and joint variables $\underline{\theta} \in R^{n}$, for kinematically redundant robots, $n>m$, and there are in general an infinite number of configurations $\underline{\theta}$ satisfying the (forward) kinematic relation $\underline{x}=f(\underline{\theta})$, for a given end effector location $\underline{x}$. This feature of redundant robot kinematics allows a "preferred" choice of configuration history $\underline{\theta}(t)$ which avoids singularities or obstacles, minimizes energy or torques, etc., while maintaining the task trajectory $\underline{x}(t)$ [4], [5]. The vast majority of approaches in the literature choose joint space trajectories (resolving the redundancy) at the velocity (rate) level, inverting the Jacobian relationship

$$
\underline{\dot{x}}=J(\underline{\theta}) \underline{\dot{\theta}}
$$

as

$$
\underline{\dot{\theta}}=\left[J_{M}\right]^{+} \underline{\dot{x}}+\left[I-J_{M}^{+} J_{M}\right] \underline{\varepsilon}
$$

using the Jacobian pseudoinverse $J_{M}^{+}$, where $J_{M}^{+}=$ $M J^{T}\left(J M J^{T}\right)^{-1}$, (with $M \in R^{n \times n}$ a positive definite matrix) is a right-sided inverse of $J$, and $\underline{\varepsilon} \in R^{n}$ is an arbitrary vector, whose selection determines which of the possible choices of configurations will be selected.

The use of the Jacobian pseudoinverse is attractive for a number of reasons. In particular, the structure of the pseudoinverse has been well investigated [6], and the behavior of solutions generated using it are convenient and well understood. In particular, the solution with $\underline{\underline{\varepsilon}}=\underline{0}$, i.e., $\underline{\dot{\theta}}=J_{M}^{+} \dot{x}$, is the solution to the problem

$$
\min \left\{\underline{\dot{\theta}}^{T} M^{-1} \dot{\dot{\theta}}\right\} \quad \text { subject to } \underline{\dot{x}}=J(\underline{\theta}) \underline{\dot{\theta}} .
$$

Numerous researchers have exploited the above property of the pseudoinverse [4], [5], choosing $M$ in various ways to synthesize "minimum energy" solutions to various problems of interest [for example, choosing $M^{-1}$ as the inertia matrix yields the solution that (locally) minimizes manipulator kinetic energy].

However, the two-norm is not the only norm with physical significance. The infinity-norm of a vector $\underline{v} \in R^{n}$ is defined as $\|\underline{v}\|_{\infty}=\max \left(\left|v_{1}\right|,\left|v_{2}\right|, \ldots,\left|v_{n}\right|\right)$. Thus for redundant manipulator inverse kinematics, the infinity-norm problem analogous to (3) above becomes

$$
\min \|\underline{\dot{\theta}}\|_{\infty} \quad \text { subject to } \underline{\dot{x}}=J(\underline{\theta}) \underline{\dot{\theta}} .
$$

Note that computing a solution by minimizing the infinity-norm yields a joint velocity vector whose maximum (absolute) joint velocity will be minimum among all vectors $\underline{\dot{\theta}}$ satisfying (1). Such a solution can thus be thought of as a minimum effort solution, as opposed to the minimum energy solution produced using the pseudoinverse [13]. 
There is strong practical motivation for such solutions-consider the common case of joint velocity (or actuator torque) limits. In this case, minimum energy (i.e., pseudoinverse-based) solutions, by their nature, can constrain only the sum of squares of the components of the solution vector, yielding no information about individual components. This is an important issue practically, since if the solution generated has an unexpected component outside the joint velocity limits, the actual trajectory implemented will be "clipped" at the corresponding joint, leading to solutions that will not in fact satisfy (1), and task space trajectories that will deviate from $\underline{x}$. This could be disastrous in many applications (consider robot surgery, for example). On the other hand, the minimum infinity norm (i.e., minimum effort) solution explicitly seeks to minimize the maximum component magnitude, thus will always find a solution satisfying the physical limit constraints if one exists. (Note that by definition, the pseudoinverse solution always has maximum component magnitude greater or equal to that of the infinity-norm solution). This is very appealing practically, suggesting that in many cases, minimum infinity-norm solutions can be tailored more effectively for physical constraints than can those using the pseudoinverse.

There have been several works investigating the use of minimum infinity-norm solutions in robotics in the last few years. The first discussion of the infinity norm in the context of robot kinematics appears to be in [20]. Minimum infinity-norm motions for open-chain manipulators via joint torque optimization has been proposed in [19], in which the minimum-infinity solution is shown to utilize energy more efficiently for several examples.

However, there are a number of problems and heretofore unresolved issues when using the infinity norm compared to the two-norm. Algorithms for computing minimum infinity norms are numerical in nature [14]-[18], and a closed form quantity paralleling the pseudoinverse has not previously been available to aid in analysis of the structure and/or computation of the solutions. In addition, unlike the case for the pseudoinverse, the question of continuity and uniqueness of minimum infinity-norm solutions has not been answered previously. This is important practically, since it has not been known when or whether the solution may become discontinuous and hence not implementable practically.

Taken together, the above restrictions and concerns have relegated the infinity-norm (a.k.a. minimum-effort) approach to something of a curiosity, since even though the results reported in the literature thus far have been promising, the general behavior of the solutions could not be guaranteed. In this paper, we introduce a new closed-form expression for an "infinity-inverse" which parallels the pseudoinverse, and use it to resolve the issues of continuity and uniqueness for the least infinity-norm solutions. The analysis resolves the difficulties mentioned above, and results in a deeper understanding of the nature of minimum infinity-norm solutions.

\section{TOWARDS DISCONTINUITY}

Before continuing on with our analysis of the behavior of least infinity norm solutions, it will be helpful to outline the precise steps we will need to take to arrive at a meaningful result. We will start with a statement of the theorem at the heart of infinity norm analysis, known as the "dual optimization theorem." Following the dual optimization theorem we review two corollaries, the "alignment corollary" and the "orthogonality corollary." The orthogonality and alignment corollaries lead to the important "equal magnitude property," and these together will help to generate a basic algorithm for finding a minimum infinity norm solution to a consistent underdetermined system. Though we will rephrase the theorem and two corollaries to suit the notation and appli- cation of manipulator redundancy, they are general and can be found in literature such as [14]-[17] and [23].

In order to discuss nonuniqueness and discontinuity, we then deviate from previous discussions on infinity-norm algorithms, and hypothesize the existence of a generalized inverse, called the "infinity inverse," which yields minimum infinity norm solutions in a closed form. Using the equal magnitude property, we extract certain properties about the infinity inverse, most notably the "Haar basis property." From there, we proceed to a discussion of the geometric meaning of least infinity-norm solutions, designed to develop the reader's intuition about the problem and answer some questions which are very difficult to tackle in abstraction. These geometric insights will allow us to discuss the nonuniqueness of least infinity norm solutions. The property of nonuniqueness opens the door to understanding when and how a discontinuity might occur in a time-varying underdetermined system. Having examined the nature of nonuniqueness and discontinuity, we then proceed to construct one possible mechanism for avoiding discontinuities by generating a metric which measures "how far away" a point of nonuniqueness is during a time-varying trajectory. We also provide several examples throughout the text to help the reader gain familiarity with various characteristics of least infinity-norm solutions.

\section{DuAl Optimization THEOREM AND COROLlaRIES}

\section{A. Dual Optimization Theorem}

Given the system $\underline{\dot{x}}=J \underline{\dot{\theta}}$ of $m$ consistent equations in $n$ unknowns $(m<n)$, then

$$
\min _{J \underline{\dot{\theta}}=\underline{\dot{x}}}\|\underline{\dot{\theta}}\|_{\infty}=\max _{\left\|J^{T} \underline{f}\right\|_{1}=1} \underline{\dot{x}}^{T} \underline{f} .
$$

There is a beautiful symmetry in the dual optimization theorem [14], [15], and at its heart we see that the minimization of the infinity norm velocity solution has been reformulated as a maximization of the endeffector work $\left(\underline{\dot{x}}^{T} f\right)$. Furthermore, the work must be maximized subject to the constraint that the associated joint torques, $\underline{\tau}=J^{T} \underline{f}$, exhibit a unit 1-norm. (It is important to realize that these are static torques and forces, introduced to aid our analysis of a purely kinematic problem. These torques and forces are not equivalent to those necessary to move the end-effector along a given trajectory.)

\section{B. Orthogonality Corollary}

For an optimal force $\underline{f}^{*}$ such that

$$
\underline{\dot{x}}^{T} \underline{f}^{*}=\max _{\left\|J^{T} \underline{f}\right\|_{1}=1} \underline{\dot{x}}^{T} \underline{f}
$$

then $m-1$ components of $J^{T} \underline{f}^{*}$ are equal to zero with the corresponding columns of $J$ linearly independent.

This corollary indicates that the optimal force maximizing the dual problem in (5) will be orthogonal to at least $m-1$ linearly independent columns of $J$, an important property as we will see later. It should be noted that more than $m-1$ components of $J^{T} f^{*}$ may be zero, but since $J \in \Re^{m \times n}$, this would imply that $f^{*}$ is orthogonal to at least $m$ columns which would indicate a degenerate case, where $J$ drops rank. We save the discussion of least infinity norm solutions near singularities for later.

\section{Alignment Corollary}

Having found the optimal $f^{*}$, the minimum infinity norm solution $\underline{\dot{\theta}}^{*} \in \ell_{\infty}$ is "aligned" with $J^{\bar{T}} f^{*} \in \ell_{1}$. That is

$$
\left[\underline{\dot{\theta}}^{*}\right]^{T}\left[J^{T} \underline{f}^{*}\right]=\left\|\dot{\hat{\theta}}^{*}\right\|_{\infty}\left\|J^{T} \underline{f}^{*}\right\|_{1} .
$$


A very important consequence of the alignment between a vectors in $\ell_{\infty}$ and $\ell_{1}$ we dub the "equal magnitude property," which will complete the background necessary to examine a basic least infinity-norm algorithm [23], [27].

\section{Equal Magnitude Property}

An optimal minimum infinity norm velocity solution, $\underline{\dot{\theta}}^{*}$, always exists with at least $n-m+1$ components of equal magnitude; furthermore, that magnitude is the maximum magnitude of all of the components of $\underline{\dot{\theta}}^{*}$.

Stated another way, where $\left\|\dot{\theta}^{*}\right\|_{\infty}=k$, then $n-m+1$ components of $\dot{\theta}^{*}$ will be equal to $\pm k$. To foreshadow future results, note that the equal magnitude property does not prohibit more than $n-m+1$ components of $\underline{\theta}^{*}$ from having equal magnitude, nor does it imply that an optimal solution must have $n-m+1$ equal magnitude components. It simply says that, except for degenerate cases, there is at least one optimal solution which will possess $n-m+1$ equal magnitude components. This will prove to be an extremely important property in the discussion of generalized inverses.

\section{INFINITY INVERSE}

In order to discuss the issue of the uniqueness of $\left\|\dot{\theta}^{*}\right\|_{\infty}$, we first imagine that there is an inverse, similar to the pseudoinverse, which will provide $\underline{\theta}^{*}$ in closed-form. Such an inverse would be one of an infinite class of "generalized inverses." For the least infinity norm problem, we adopt a general form for inverse

$$
J^{\#}=Q(J Q)^{-1}, \quad Q \in \Re^{n \times m} .
$$

Setting $Q=J^{T}$ yields the pseudoinverse, so adopting the form in (8) would seem intuitively "good." However, the obvious problem remains to quantify, if possible, the construction of $Q$. Just as with the pseudoinverse in this form, we require full rank for $J$ and $Q$.

The choice for $J^{\#}$ in (8) is so general, in fact, that any choice of $Q$ for which $(J Q)^{-1}$ exists will generate a solution. This can be seen by simply observing that, if $\underline{\dot{\theta}}=Q(J Q)^{-1} \underline{\dot{x}}$, then $J \underline{\dot{\theta}}=J Q(J Q)^{-1} \underline{\dot{x}}=$ $\underline{\dot{x}}$. In general, there seems to be no obvious method to produce the $Q$ which generates any given $\underline{\dot{\theta}}$, but in the specific case of least infinity norm solutions, but a little bit of logic and judicious use of the equal magnitude property yields the following simple rules for constructing $Q$ given $\dot{\theta}^{*}$ from one of the algorithms in [14]-[16].

2) Pick some index $k$ such that $\left|\underline{\theta}^{*}(k)\right|=\left\|\underline{\theta}^{*}\right\|_{\infty}$ (there will be at least $n-m+1$ choices). Set

$$
[k \text { th row of } Q] \triangleq Q(k)=[1,1, \ldots, 1] \in \Re^{m} \text {. }
$$

3) For rows $i$ where $\left|\dot{\theta}^{*}(i)\right|=\left|\underline{\theta}^{*}(k)\right|$

$$
Q(i)=\left(\operatorname{sign}\left[\underline{\dot{\theta}}^{*}(i)\right] \cdot \operatorname{sign}\left[\underline{\dot{\theta}}^{*}(k)\right]\right) Q(k) \text {. }
$$

If there are more than $n-m+1$ equal magnitude elements of $\underline{\dot{\theta}}^{*}$, stop after determining $n-m+1$ rows.

4) All other rows (the remaining $m-1$ ) must be linearly independent (a good choice will be presented shortly). If there are more than $n-m+1$ equal magnitude elements of $\underline{\theta}^{*}$ and $(J Q)^{-1}$ does not exist, return to step 2 and choose a different set of rows in expression (10).

We should note several items regarding the algorithm above. First, the rows of ones assigned in (9) is arbitrary, though as we shall see shortly, very meaningful. Second, the algorithm assumes full rank $J$, as without that condition, $(J Q)^{-1}$ cannot exist under any circumstance. Third, by design $Q$ must have full rank. Also, the algorithm as such does not construct a "feed-forward" inverse; that is, $\dot{\theta}^{*}$ must already be known (from an algorithm like Cadzow [14]) in order to infer a $Q$ which would generate it in closed from. At first glance this might seem of limited utility, however remember that we have not designed this inverse as a computational tool but rather an analysis tool.

While $n-m+1$ rows of $Q$ are either a positive or negative row of ones, $m-1$ rows remain to be assigned in step 3. An interesting choice would be to pick from the set of $m$-vectors whose components are \pm 1 . The cardinality of this set is $2^{m}-2$, more than enough for the $m-1$ remaining rows. (Rows of all 1 or -1 are already used to construct $Q$ in the recipe above.) Basis vectors consisting of 1's and -1 's are generically known as "Haar" basis vectors, and concept of the Haar basis is very powerful in fields like wavelet analysis. Naturally, if the rows of $Q$ consist of a Haar basis subset, then the columns of $Q$ must also consists of a (higher dimensional) Haar basis subset, which we term the "Haar basis condition."

\section{A. Haar Basis Condition.}

To satisfy an infinity inverse $\underline{\theta}^{*}=Q(J Q)^{-1} \underline{\dot{x}}$, a (nonunique) $Q$ may be constructed whose linearly independent columns are a subset of the extended Haar basis set, $\left.\left\{\begin{array}{llll} \pm 1 & \pm 1 & \cdots & \pm 1\end{array}\right]^{T}\right\} \subset \Re^{n}$.

At this point, it may be instructive to see an example. Take the randomly generated matrix $J$ as

$$
J=\left[\begin{array}{llll}
0.4660 & 0.8462 & 0.2026 & 0.8381 \\
0.4186 & 0.5252 & 0.6721 & 0.0196
\end{array}\right] .
$$

Now take $\underline{\dot{x}}$ as

$$
\underline{\dot{x}}=\left[\begin{array}{r}
1 \\
-2
\end{array}\right] .
$$

Computing $\underline{\dot{\theta}}^{*}$ via the Cadzow algorithm gives

$$
\underline{\theta}^{*}=\left[\begin{array}{r}
-2.2278 \\
0.7356 \\
-2.2278 \\
2.2278
\end{array}\right] \text {. }
$$

(Note the $n-m+1=3$ equal magnitude components.) Following the rules to construct an inverse, let the first row of $Q$ be $[1,1]$ (i.e., the index $k=1$ ). Then the third and fourth rows must be $[1,1]$ and $[-1,-1]$. The second row must simply be linearly independent of everything else, so a candidate $Q$ is

$$
Q=\left[\begin{array}{rr}
1 & 1 \\
1 & -1 \\
1 & 1 \\
-1 & -1
\end{array}\right]
$$

Now, the usual infinity inverse relation holds

$$
\underline{\dot{\theta}}^{*}=Q(J Q)^{-1} \underline{\dot{x}}
$$

and $\underline{\dot{\theta}}^{*}$ will be the solution of minimal maximum magnitude.

\section{GEOMETRIC VIEW}

We now introduce a geometric illustration of the mathematics behind infinity norms. At this point, a few images combined with the information presented in the previous sections will help to clear up issues such as the seemingly arbitrary choice of the Haar basis columns for $Q$. The geometric arguments also provide exactly the right framework for discussing nonuniqueness and discontinuity, and we have found them quite intuitively satisfying. 


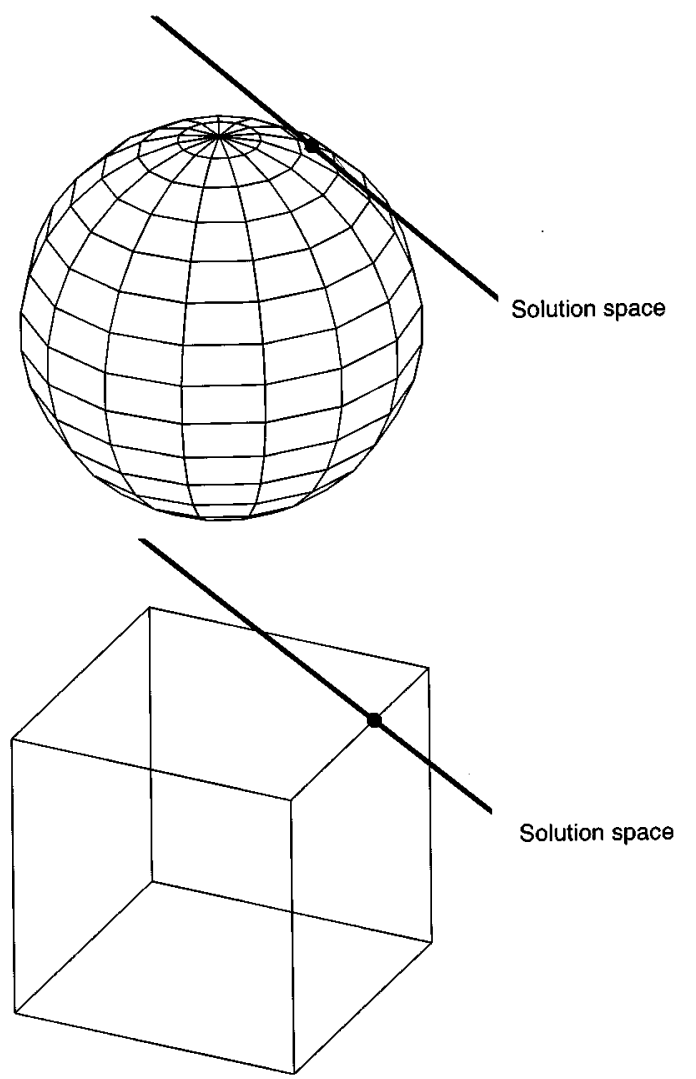

Fig. 1. Here are the two-norm and $\infty$-norm equinorm surfaces in $\Re^{3}$. The solution set $\mathcal{S}$ is one-dimensional, and it is clear exactly where the equinorm surfaces intersect $\mathcal{S}$ (the minimum norm solutions).

Imagine the set $\mathcal{S}$ of all possible solutions

$$
\mathcal{S} \triangleq\{\underline{\dot{\theta}}: J \underline{\dot{\theta}}=\underline{\dot{x}}\}
$$

Because the constraint equations $J \underline{\dot{\theta}}=\underline{\dot{x}}$ are linear, $\mathcal{S}$ will take the form of a line, a plane or in general a hyperplane in $n$ dimensions. Now imagine an "equinorm" surface $\mathcal{B}(r, p)$, defined by all the points

$$
\mathcal{B}(r, p) \triangleq\left\{\underline{z}:\|\underline{z}\|_{p}=r\right\}, \quad \underline{z} \in \Re^{n}
$$

where $r>0$ is known as the equinorm radius. The shape of the equinorm surface is extremely important in determining the characteristics of a minimum $p$-norm solution for $\underline{\dot{\theta}}$. If the equinorm surface starts as a point $(r=0)$ and gradually grows ( $r$ gets larger and larger) then eventually a point is reached where the equinorm surface just intersects the solution set. The point of intersection is a minimum $p$-norm solution, $\dot{\theta}^{*}$.

This situation can be seen clearly in Fig. 1, which would represent the case that $J \in \Re^{2 \times 3}$. The equinorm surfaces intersect the solution space at a unique point, the minimum norm solution for two-norm and infinity-norm domains, respectively.

At this point, it is instructive to see why the notion of joint variable constraints fits so nicely with this geometric picture. Since we are solving a velocity kinematics problem, the constraints of interest would be maximum joint speeds. (As in [10] and [19] the problem may be reformulated to optimize generalized static forces or torques, respectively, in which case the constraint sets would represent maximum dynamic torques or maximum static forces achievable by the actuators.)
A natural way to formulate a constraint set, called $\mathcal{C}_{0}$ here, would be

$$
\mathcal{C}_{0}=\left\{\dot{\theta}_{i}:-k_{i} \leq \dot{\theta}_{i} \leq k_{i}\right\}, \quad i=1,2, \ldots, n
$$

and note that $\mathcal{C}_{0} \subseteq \Re^{n}$, and takes the shape of a polytope aligned with the elementary basis vectors $[1,0,0, \ldots, 0],[0,1,0, \ldots, 0]$, $\ldots,[0,0, \ldots, 1]$. (If some actuator constraints are dependent, then the polytope may not align with certain basis directions.) If we make a variable substitution, $\underline{\dot{\theta}}=D \underline{\dot{\theta}}$, in the vast majority of cases, there exists a nonsingular weighting matrix $D$ such that we may normalize polytope $\mathcal{C}_{0}$ into a new constraint set $\mathcal{C}_{1}$ with

$$
\mathcal{C}_{1}=\left\{\tilde{\dot{\theta}}_{i}:-k \leq \tilde{\dot{\theta}}_{i} \leq k\right\}, \quad i=1,2, \ldots, n
$$

Letting $\tilde{J}=J D^{-1}$, the optimization problem can be solved in normalized space, and the solution then de-normalized at the end, $\dot{\theta}^{*}=$ $D^{-1} \tilde{\tilde{\theta}}^{*}$. The important observation here is that the weighting matrix $D$ "stretches" and "squashes" the original constraint polytope into a hypercube such that

$$
\mathcal{B}(r, \infty) \subseteq \mathcal{C}_{1} \quad \forall r \leq k
$$

If the joint constraints are not to be exceeded, the solution space $\mathcal{S}$ (in general, a "hyperplane") must intersect $\mathcal{C}_{1}$, otherwise no solutions exist which do not exceed the constraint conditions. In general, an infinite number of solutions may in fact exist which intersect the constraint hypercube, including the minimum infinity-norm solution. Recall that the least infinity-norm solution $\tilde{\hat{\theta}}^{*}$ can be found by "growing" a hypercube surface $\mathcal{B}(r, \infty)$ from $r=0$ until the very first point where $\{\mathcal{B}(r, \infty) \cap \mathcal{S}\} \neq \phi$. Remembering that $\left\|\underline{\tilde{\theta}}^{*}\right\|_{\infty}=r$, and using the hypercube constraint relation in (20), we may deduce one of the most useful and important properties of minimum infinity-norm solutions:

\section{A. Optimal Constraint Property}

For a solution $\underline{\theta}^{*}$ obtained using a normalized least infinity norm (as above), if any element of $\underline{\dot{\theta}}^{*}$ exceeds the associated joint constraint, then no solution exists which will not exceed the constraint set.

To see this, assume that a least infinity norm solution $\underline{\theta}^{*}$ does not satisfy a particular constraint set $\mathcal{C}_{1}$; in this case the minimum equinorm hypercube surface $\mathcal{B}(r, \infty)$ is "bigger" than $\mathcal{C}_{1}$ (i.e., $r>k$ ). If an solution $\underline{\dot{\theta}}_{\text {alt }} \in \mathcal{S}$ exists which does satisfy the constraints, then solution space $\mathcal{S}$ must intersect $\mathcal{C}_{1}$ at some point. However, since $\mathcal{B}(r, \infty)$ surrounds $\mathcal{C}_{1}$, then $\mathcal{B}(r, \infty)$ must not be the smallest hypercube which intersects $\mathcal{S}\left(\mathcal{C}_{1}\right.$ is smaller), implying that $\underline{\theta}^{*}$ is not the least infinity norm solution.

Henceforth, we will assume that the problem has already been normalized (or does not need to be normalized) in order to simplify notation.

\section{NONUNIQUENESS}

At last we are in a position to discuss the issue of nonuniqueness. Again consider the case of a minimum two-norm solution. Because a sphere is "round," and $\mathcal{S}$ for a set of linear equations must be convex and complete (all elements and limit points of the set are linear combinations of other points in the set), as the hypersphere grows from the origin, there will be one point where the sphere uniquely intersects the solution space $\mathcal{S}$. This is the intuitive reason why minimum two-norm solutions are always unique. However, it is possible for $\mathcal{S}$ to align itself to be "parallel" to a face of the infinity-norm hypercube. In this case, as $r$ grows, the intersection set $\{\mathcal{B}(r, \infty) \cap \mathcal{S}\}$ has infinite cardinality! 


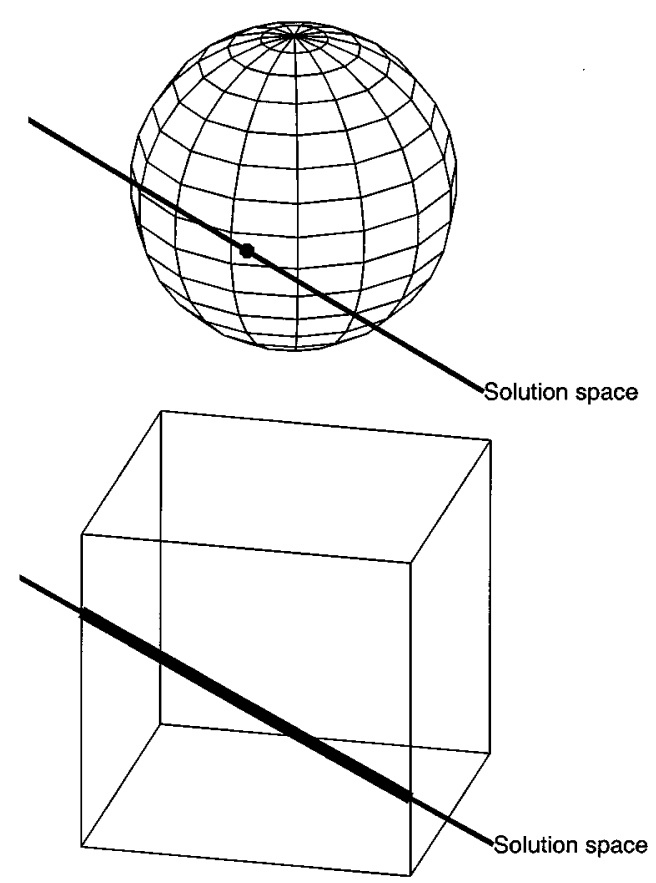

Fig. 2. Here, while the intersection of the sphere and $\mathcal{S}$ remains unique, the cube intersects $\mathcal{S}$ allowing an infinite number of minimum infinity-norm solutions.

An infinite number of possible solutions, all lying on the face of the hypercube, are allowable. They are all minimum infinity-norm solutions, and this is a point of nonuniqueness. Fig. 2 illustrates this phenomenon, with the thick line representing all of the allowable portion of $\mathcal{S}$ which consists of minimum norm solutions.

From the pictures, one can get the intuitive sense that, as the equinorm hypercube $\mathcal{B}(r, \infty)$ grows, it will first intersect with solution space $\mathcal{S}$ at some edge, or at the worst case, along a hypercube face. It would seem natural to represent a hypercube with a list of its vertices, which number $2^{n}$ and take the form

$$
\text { vertices }=\left\{\left[\begin{array}{c}
r \\
r \\
\vdots \\
r \\
r
\end{array}\right]\left[\begin{array}{r}
r \\
r \\
\vdots \\
r \\
-r
\end{array}\right]\left[\begin{array}{r}
r \\
r \\
\vdots \\
-r \\
r
\end{array}\right] \cdots\left[\begin{array}{c}
-r \\
-r \\
\vdots \\
-r \\
-r
\end{array}\right]\right\} \text {. }
$$

Thus, where $J \in \Re^{m \times n}$, it will require a linear combination of a minimum of $m$ vertices to represent the point of intersection, $\underline{\theta}^{*}$. Labeling those vertices as column vectors $\underline{v}_{1}, \ldots, \underline{v}_{m}$, we then write, for some appropriate $\underline{c} \in \Re^{m}$

$$
\underline{\theta}^{*}=\left[\underline{v}_{1}, \ldots, \underline{v}_{m}\right] \cdot \underline{c}=r\left[\begin{array}{cccc} 
\pm 1 & \pm 1 & \cdots & \pm 1 \\
\pm 1 & \pm 1 & \cdots & \pm 1 \\
\vdots & \vdots & & \vdots \\
\pm 1 & \pm 1 & \cdots & \pm 1
\end{array}\right] \cdot \underline{c} .
$$

We now recall the general form for the optimal infinity inverse, $\underline{\dot{\theta}}^{*}=\hat{Q}(J \hat{Q})^{-1} \underline{\dot{x}}$, and set this equal with expression (22). Now, $\left[\underline{v}_{1}, \ldots, \underline{v}_{m}\right]=\hat{Q}$, and we may simplify so that

$$
\begin{aligned}
\underline{\dot{\theta}}^{*} & =\left[\underline{v}_{1}, \ldots, \underline{v}_{m}\right] \underline{c}=\hat{Q}(J \hat{Q})^{-1} \underline{\dot{x}} \\
& =Q r(J Q r)^{-1} \underline{\dot{x}}=Q(J Q)^{-1} \underline{\dot{x}}
\end{aligned}
$$

where $Q$ is the matrix of ones and minus ones listed in (22). This explains the reasoning for choosing $Q$ to have rows of \pm 1 as we did in the algorithm for the construction of $Q$ in the section on the infinity inverse. In general, $r$ need not merely be a scalar, but could be any full rank $m \times m$ postmultiplier, because the only requirements of $Q$ are that it have full rank and the solution $\underline{\theta}^{*}$ is contained within the column space of $Q$. (In other words, for any full rank $T \in \Re^{m \times m}$, if $\hat{Q}=Q T$ then $\operatorname{col}$ space $\{\hat{Q}\}=\operatorname{col}$ space $\{Q\}$.) The case of a nonunique optimal solution, however, presents the interesting situation that the column space of $Q$ is not enough to represent all of the optimal solutions. Multiple valid infinity inverses will exist with $Q$ 's which span different spaces. This is exactly what we expect if the solution space lies on the face of a hypercube - a particular solution may be a linear combination of more than the minimally required $m$ hypercube vertices.

Again, a simple example may help to solidify the reader's intuition. Take the system $J \underline{\dot{\theta}}=\underline{\dot{x}}$ as

$$
\left[\begin{array}{rrrr}
2 & -2 & -1 & -1 \\
5 & 3 & 1.5 & 1.5
\end{array}\right] \cdot \underline{\dot{\theta}}=\left[\begin{array}{l}
2 \\
1
\end{array}\right]
$$

As necessary, $J$ has full rank. One optimal solution $\underline{\theta}^{(1)}=$ $\left[\begin{array}{llll}0.5 & -0.5 & -0.5 & 0.5\end{array}\right]^{T}$, which is given by the combination of vertices

$$
Q^{(1)}=\left[\begin{array}{rr}
1 & 1 \\
-1 & -1 \\
1 & -1 \\
1 & 1
\end{array}\right]
$$

A simple computation will reveal that the solution $\underline{\hat{\theta}}^{(2)}=$ $\left[\begin{array}{llll}0.5 & -0.5 & 0.5 & -0.5\end{array}\right]^{T}$ is also valid, and possesses minimum infinity norm. The infinity inverse for this solution has

$$
Q^{(2)}=\left[\begin{array}{rr}
1 & 1 \\
-1 & -1 \\
1 & 1 \\
-1 & 1
\end{array}\right]
$$

and one can see by observation that $\operatorname{colspace}\left\{Q^{(1)}\right\} \neq$ colspace $\left\{Q^{(2)}\right\}$. In fact, the pseudoinverse itself yields $\underline{\dot{\theta}}^{(3)}=\left[\begin{array}{llll}0.5 & -0.3333 & -0.1667 & -0.1667\end{array}\right]^{T}$ which is also a valid least infinity-norm solution.

\section{DISCONTINUITY}

Having developed a sense for why least infinity norm solutions may be nonunique, and disproved solution uniqueness by example, we now proceed to discuss the problem of discontinuity in a time-varying underdetermined system. Essentially, the possibility of a discontinuity in the system exists purely because of the possibility for nonuniqueness: if the system trajectory orients the solution space $S$ so that it is "parallel" to a hypercube face, the solution may jump from one edge to the other (i.e., from $\underline{\dot{\theta}}^{(1)}$ to $\underline{\dot{\theta}}^{(2)}$ in the previous example) before continuing smoothly on its way. It should noted however, that reaching a point of nonuniqueness is only a necessary condition to experience a discontinuity, not sufficient, because the system trajectory may not actually complete the jump, choosing instead to reverse course or "veer away."

Before continuing, we should note that our discussion of solution discontinuity assumes that the system Jacobian $J(\underline{\theta}(t))$ varies smoothly with time (true for all usual robot Jacobians), and that the desired system input $\underline{\dot{x}}$ also varies smoothly. We begin by making the qualitative observation that, after the system moves around a bit, at some point the column span of $Q$ in the infinity inverse will have to change; it cannot remain constant over all trajectories. Since we know 


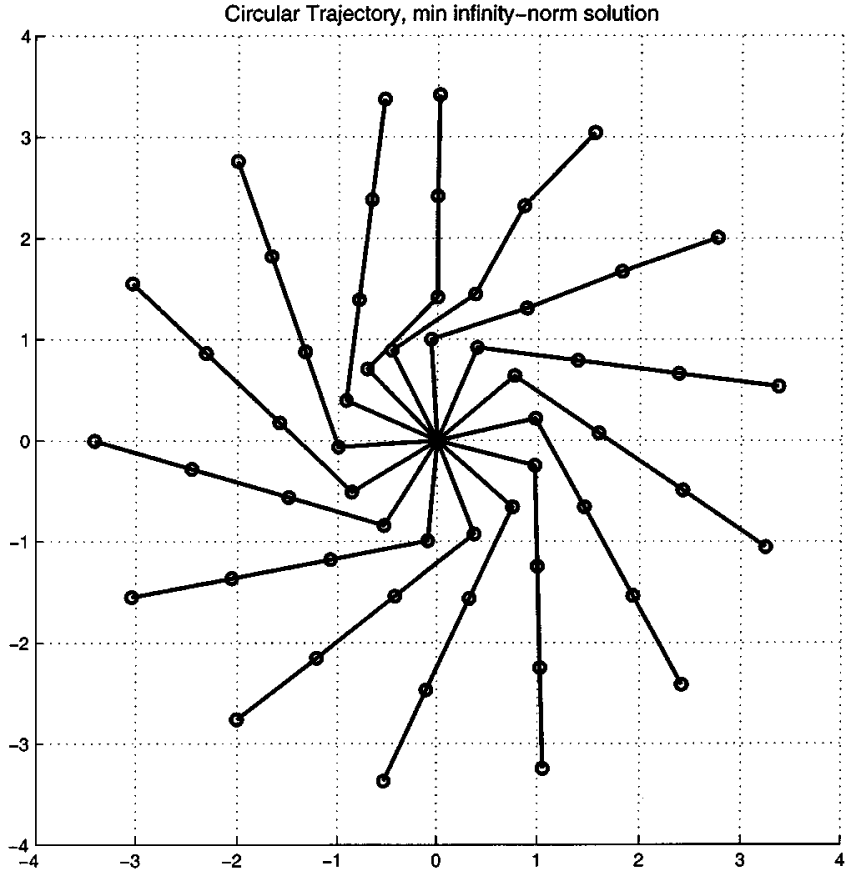

Fig. 3. The robot starts out in the initial configuration [235, -90, 45, 0] (pointing vertically) in $\mathrm{CCW}$ degrees from the previous link. The robot executes a CW circular trajectory.

that it can always be constructed of \pm 1 's, we infer that when one or more columns of $Q$ change, they do so abruptly and discontinuously. (In other words, the space spanned by the columns of $Q$ will abruptly change.) If the system output $\underline{\dot{\theta}}$ is to remain continuous during a change of the column space, then $\underline{\dot{\theta}}$ must be independent of the columns which experience the change at the instant in time when the change occurs.

Consider four-link planar robot executing a circular trajectory, as in Fig. 3. A circular trajectory about the first joint will, by its very nature, force the distal joints $(2,3$, and 4$)$ to zero velocity because only joint 1 need move to maintain a constant end-effector radius. While the simulated robot behaves very similarly for both the minimum two-norm and minimum infinity-norm algorithms, Figs. 4 and 5 illustrate the real story. Clearly the minimum two-norm solution allows the speed of joints 2, 3, and 4 to settle around zero, as expected. However, given that efficient least-infinity norm algorithms are based on the equal magnitude property, the only way to approximate zero net motion in a joint is to slam the desired joint speeds between the maximum positive and negative allowable values as fast as possible (in this case, at the update rate). Clearly the circle trajectory, by design, forces discontinuous behavior in the least infinity-norm algorithm.

As per the earlier discussion on nonuniqueness, during the discontinuity there are alternate solutions which will accomplish the endeffector trajectory-namely the solution to which the pseudoinverse tends $\left(\underline{\dot{\theta}}=[-0.78,0,0,0]^{T}\right)$. However, the least-infinity norm solution would not be able to smoothly decay the speeds of joint 2,3 , and 4 to zero as with the pseudoinverse; in order to choose that particular solution the least infinity norm algorithm would have to discontinuously jump there and remain there. It would seem there is no way to avoid at least one discontinuity during the circle trajectory using an unadulterated least infinity norm solution.

\section{AvoIding DiscontinUITIES}

Now that discontinuities are known to exist, the natural question to ask is how to avoid them. One possibility would involve storing a small number of sequential solutions in a buffer, and detecting when
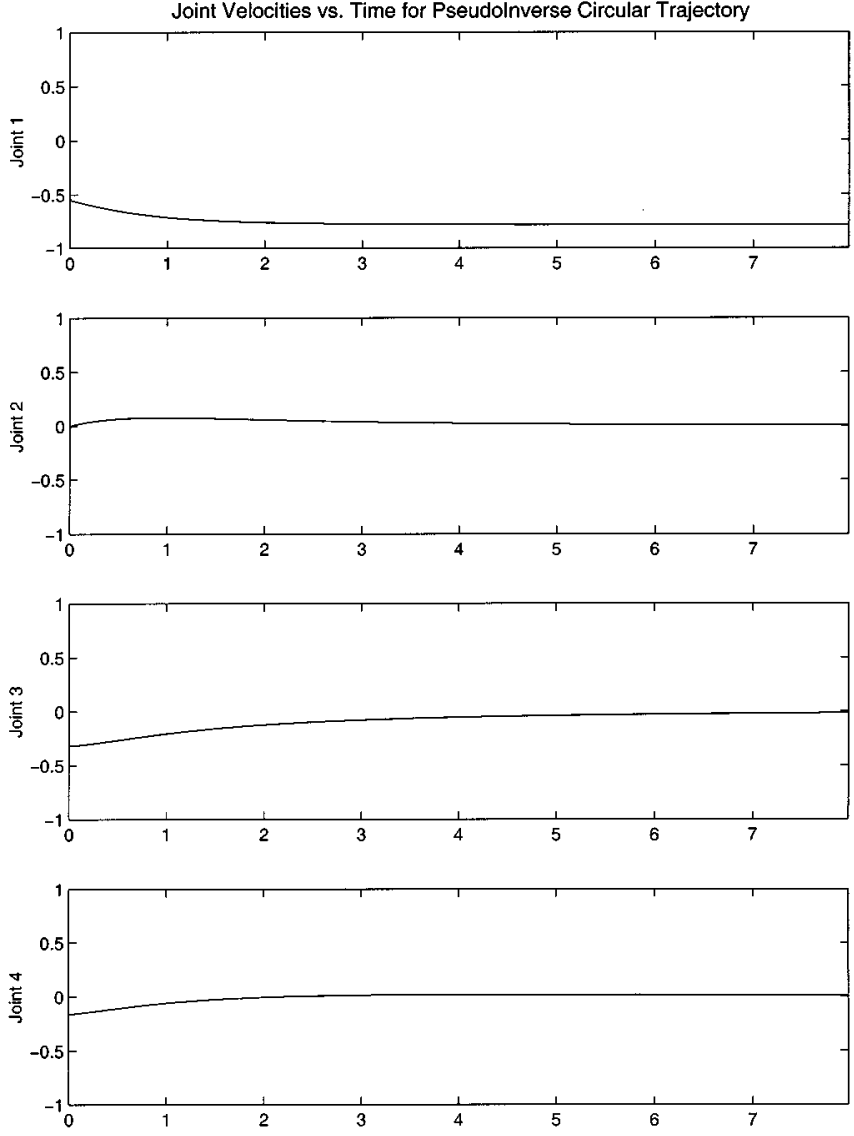

Fig. 4. The joint velocities for the circular trajectory using the pseudoinverse. The speed of joints 2,3 , and 4 drops to zero.

the difference between the next computed solution and the previous solution exceeds a certain threshold. Then, solutions already in the buffer could be modified to smoothly switch from the old trajectory to the new. However, this method degrades one of the golden properties of just-in-time (local) optimization, the ability to react to changing environments. Because the procedure now contains a "delay," the system cannot react instantly to changes in the input.

To repair the discontinuity problem, we propose a predictive method using a metric which "senses" when a discontinuity is near, and gradually takes corrective action. We dub the method "rate mixing," [2] and it has the following form:

$$
\dot{\dot{\theta}}^{*}=r \underline{\dot{\theta}}^{(\infty)}+(1-r) \underline{\dot{\theta}}^{(2)}, \quad 0 \leq r \leq 1
$$

We denote the optimal (final) solution as $\underline{\hat{\theta}}^{*}$, the least infinity-norm solution as $\underline{\dot{\theta}}^{(\infty)}$ and the least two-norm solution as $\underline{\dot{\theta}}^{(2)}$. Essentially, expression (27) "mixes" the optimal infinity-norm solution with another solution known to be continuous but suboptimal (in this case, the pseudoinverse solution, though any suboptimal continuous solution will work). Conceptually, we now want to vary this parameter $r$, called the "mixing factor," so that it stays near 1 most of the time, moving to 0 at points of discontinuity. Under these constraints, if $r$ varies continuously, $\underline{\dot{\theta}}^{*}$ will vary continuously because $\underline{\dot{\theta}}^{(2)}$ is always unique and continuous. The choice of a good mixing factor depends on several conditions which we explore next.

From a geometric point of view, recall that finding $\underline{\dot{\theta}}^{(\infty)}$ involves "growing" the hypercube [i.e., increasing $k$ in (19) from 0] until one edge of it touches the solution space $\mathcal{S}$ [19], where $\mathcal{S}=\{\underline{\dot{\theta}}: J \underline{\dot{\theta}}=\underline{\dot{x}}\}$ and $\mathcal{S}$ is always parallel to the nullspace of $J$, denoted $\mathcal{N}$. Note $\mathcal{N} \in$ 

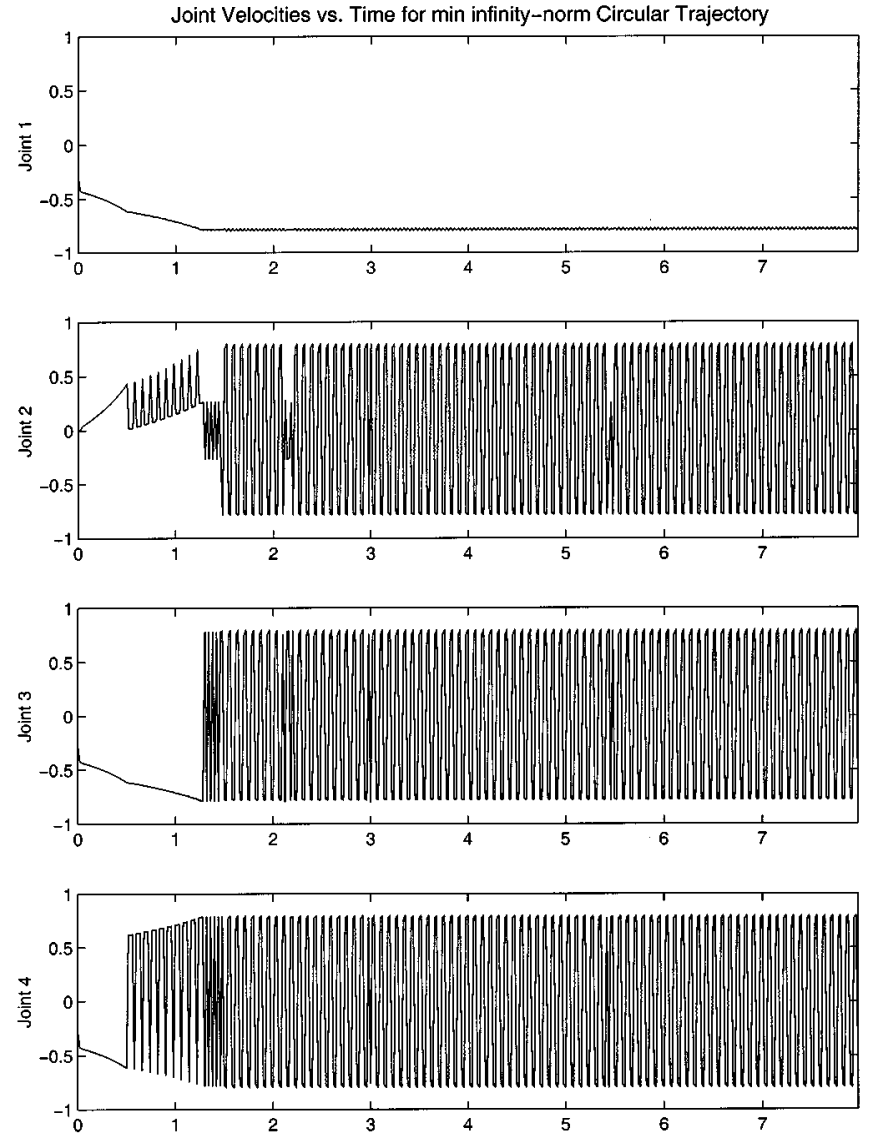

Fig. 5. Joint velocities of the circle trajectory using infinity-norm procedures. Because of the equal-magnitude property, the speed of joints 2, 3, and 4 never approach zero, but oscillate around zero.

$\Re^{n-m}$, and we will represent the nullspace with a matrix $N$ such that the orthogonal columns of $N$ span the nullspace. In other words, let

$$
N \in \Re^{n \times(n-m)}: \operatorname{span}\left\{\underline{n}_{1}, \underline{n}_{2}, \ldots, \underline{n}_{n-m}\right\}=\mathcal{N} \text {. }
$$

As the hypercube expands, just where it first intersects the solution space is the point $\underline{\dot{\theta}}^{(\infty)}$ (refer back to Figs. 1 and 2). If the nullspace is exactly aligned with a side or an edge of the hypercube, then $\{\mathcal{B}(r, \infty) \cap \mathcal{S}\}$ contains an infinity of solutions, representing the case of a nonunique optimal solution $\underline{\dot{\theta}}^{(\infty)}$. This situation, which we will call the "zero subspace angle condition," will prove useful, so we now proceed to define this condition more rigorously.

The subspace angle is defined geometrically as the angle between two hyperplanes (subspaces $\mathcal{S}_{1}$ and $\mathcal{S}_{2}$ ) embedded in a higher dimensional space. For instance, if $\operatorname{span}\left\{[0,1,0]^{T}\right\}=\mathcal{S}_{1}$ and $\operatorname{span}\left\{[0,(1 / \sqrt{2}),(1 / \sqrt{2})]^{T},[1,0,0]^{T}\right\}=\mathcal{S}_{2}$, then subspace $\left(\mathcal{S}_{1}, \mathcal{S}_{2}\right)=45^{\circ}$. More precisely, let $S_{1}$ be a matrix with orthogonal columns which span $\mathcal{S}_{1}$, and $S_{2}$ be a matrix with orthogonal columns which span $\mathcal{S}_{2}$. Then

$$
\operatorname{subspace}\left(\mathcal{S}_{1}, \mathcal{S}_{2}\right)=\cos ^{-1}(\sigma), \quad \sigma=\min \{\operatorname{diag}(\Sigma)\}
$$
with

$$
U \Sigma V^{T}=S_{1}^{T} S_{2}
$$

The subspace angle is the inverse cosine of the minimum singular value of $S_{1}^{T} S_{2}$ [22].

This is relatively unwieldy, but its use will be simplified shortly. Conceptually, we want to know if the solution space $\mathcal{S}$ is "parallel" to any face of the hypercube in $n$-space. While Figs. 1 and 2 involve only a one-dimensional solution space in $\Re^{3}$, in general the solution space is multidimensional in $\Re^{n}$ and may be parallel not just to a $(n-1)$-dimensional face of the hypercube, but any subfaces or edges of it as well. This generalization makes the problem significantly harder, and is the reason we investigate the concept of the subspace angle.

We begin by assigning $S_{1}=N$. (Because $\mathcal{S}$ and $\mathcal{N}$ are parallel linear spaces, the subspace angle will be the same whether the columns of $S_{1}$ span the solution space or the nullspace). Since we are concerned with the solution space intersecting edges and faces of the hypercube (which are always aligned with the elementary basis vectors), we will choose $m$ of these basis vectors to be the columns of $S_{2}$, so that, if $\mathcal{N}$ and $\mathcal{S}_{2}$ are independent spaces, $\mathcal{N} \cup \mathcal{S}_{2}=\Re^{n}$. That is, if the spaces are independent, then their union should be the complete space $\Re^{n}$. Now the question is, which $m$ basis vectors should span $\mathcal{S}_{2}$ ? There will be $\left(\begin{array}{l}n \\ m\end{array}\right)$ possible arrangements and, from the above discussion, we want to know if the nullspace has a zero subspace angle with any one of those choices. Now we note the following result.

\section{A. Zero Subspace Angle Condition}

For an underdetermined system employing least infinity norm optimization with an optimum solution $\underline{\theta}^{(\infty)}$, if $\underline{\theta}^{(\infty)}$ is nonunique then there exists some $\mathcal{S}_{2}$ spanned by elementary basis vectors such that $\cos ^{-1}\left(\sigma_{1}\right)=0$.

Finding the subspace angle for $\left(\begin{array}{l}n \\ m\end{array}\right)$ subspaces could take a long time using the singular value decomposition method, and yields little intuition. Since we do not need the actual angle, only a number representative of the angle (in a one-to-one correspondence) we may note that, by filling a $n \times n$ matrix with columns which span the two subspaces, a zero determinant indicates that the column space is not complete. Since we know that, by construction, $S_{2}$ consists of $m$ columns of 0's and 1's (no column repeating), it will always span $\Re^{m}$. Recall that we must consider all possible choices of $S_{2}$. Therefore, to find the minimum subspace angle over all possible faces and edges of the hypercube (all possible $S_{2}$ ), we may concatenate $N$ and $S_{2}$ into a $n \times n$ matrix and find its determinant. A zero determinant corresponds to a zero minimum subspace angle; larger absolute value determinants indicate larger subspace angles. Note that we may also swap rows in this concatenation matrix in the following manner:

$$
\left[N \mid S_{2}\right] \rightarrow\left[\begin{array}{cc}
N_{1} & 0 \\
N_{2} & I_{m \times m}
\end{array}\right] \quad \forall S_{2}
$$

with the result that

$$
\left|\operatorname{det}\left[N \mid S_{2}\right]\right|=\left|\operatorname{det}\left[N_{1}\right] * \operatorname{det}[I]\right|=\left|\operatorname{det}\left[N_{1}\right]\right| .
$$

Now $\left(\begin{array}{l}n \\ m\end{array}\right)$ subspace angle calculations have been distilled down to $\left(\begin{array}{l}n \\ m\end{array}\right)$ determinants of size $(n-m) \times(n-m)$. Define

$$
d_{\min }=\min _{\mathrm{S}_{2}}\left\{\left|\operatorname{det}\left[N_{1}\right]\right|\right\} .
$$

In an evolving trajectory, if $d_{\min }$ approaches zero, the solution $\underline{\dot{\theta}}^{(\infty)}$ gets closer and closer to a point of nonuniqueness, and possible discontinuity. Note $d_{\min }$ is continuous (although not smooth) if the elements of $N$ vary continuously; this implies the elements of $J$ varying continuously - a reasonable expectation if the input trajectory contains no discontinuities.

Only one step remains to refine $d_{\text {min }}$ into the mixing factor $r$ from (27). We must limit $r$ to exist only between 0 and 1, which can be accomplished as

$$
r=1-e^{-a \cdot d_{\min }} .
$$



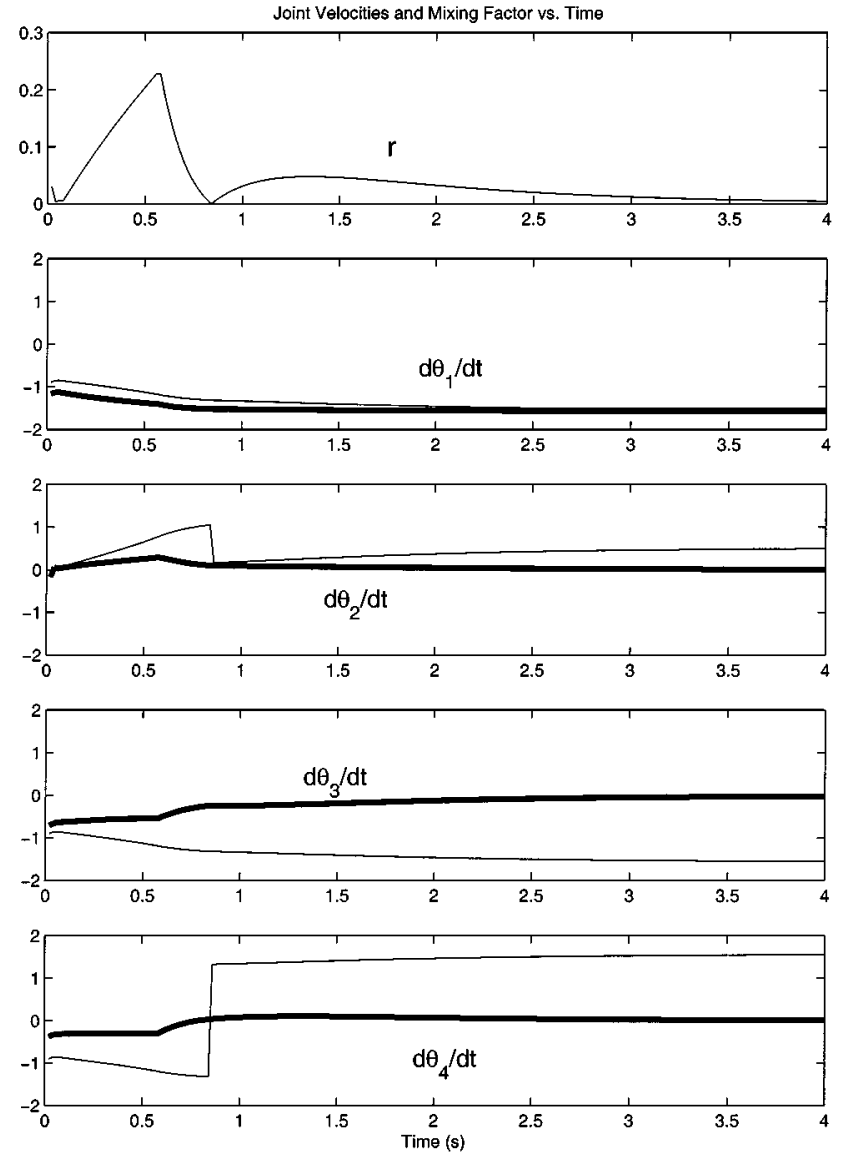

Fig. 6. Plots of the mixing factor and the joint velocities of the corrected circle example. Thick lines are trajectories corrected for discontinuities; thin lines represent what a pure least infinity-norm solution would have produced.

Recall that $d_{\min }>0$. The factor $a$ determines how quickly $\underline{\theta}^{*}$ will switch from a least infinity norm solution to a two-norm solution, tending to "round out" the sharp edges of the hypercube.

The rate mixing method has proven quite effective in a number of examples, including the circle example, for which the mixing factor and joint velocities appear in Fig. 6 . The first plot shows how $r$ varies with time, heavily favoring the pseudoinverse solution to avoid the oscillations present from Fig. 5. Also shown is a linear trajectory, in Fig. 7, specifying $\underline{\dot{x}}=[-0.8,-0.8]$ for about $4 \mathrm{~s}$. Note that this trajectory contains an isolated discontinuity which the robot smoothly avoids, favoring the infinity norm solution most of the time. Also note that the mixing factor hits zero once where there is no discontinuity. This represents a point of nonuniqueness, however the robot's trajectory did not take it "across" the nonunique point to create a discontinuity. This instance reflects the necessary, but not sufficient, quality of the mixing factor and would seem to be inevitable from time to time without apriori knowledge of the future trajectory.

Although the general algorithm requires a search for the minimum determinant of all $(n-m) \times(n-m)$ minors of $N$, for most rigid-link robots, the typical degree of redundancy will be only one or two. In the most common case-one degree redundant - the determinants in (32) boil down to a simple search for the smallest magnitude element of the nullspace vector. This should make intuitive sense. For example, in three dimensions, if the $z$ element of a one-dimensional nullspace vector equals zero, then that vector must be parallel to the $x-y$ plane, a necessary condition for discontinuity in least infinity-norm problems. Given this fact, if the Jacobian nullspace vector exists in closed form, determining if and when any component goes to zero ought to provide
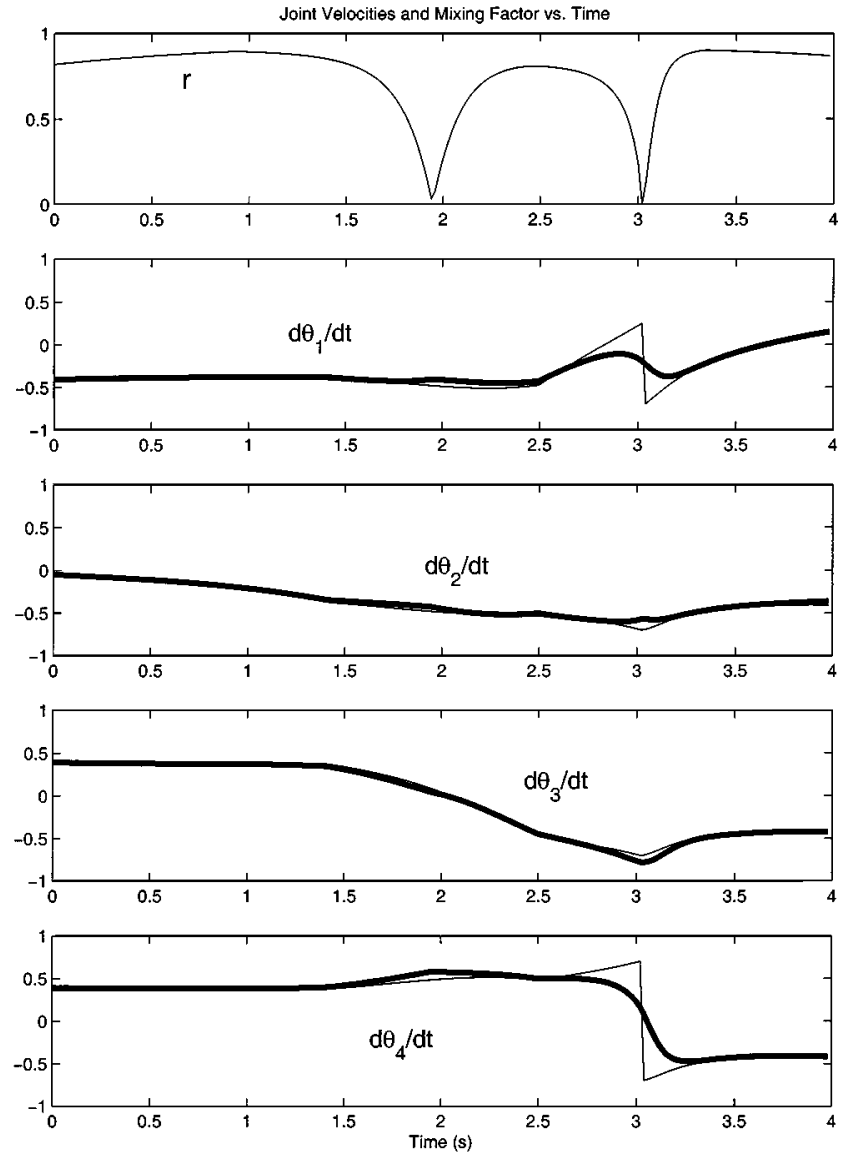

Fig. 7. A corrected linear trajectory. Note how the rate-mixing algorithm successfully avoids one discontinuity, but also suffers one "false alarm."

insight into the conditions when the manipulator might possibly reach points of nonuniqueness.

\section{CONCLUSIONS}

Unfortunately, space does not permit the further examination of details like behavior of the infinity inverse near singularities and examination of possibilties that the product $J Q$ might not have full rank. However, these details do not materially affect the observations and algorithms in this paper, and they can be found in [3].

We have endeavored in this paper to illustrate and explore many details and subtleties of infinity norms and least effort solutions in general, using kinematic redundancy resolution as a test case for the mathematical theory. After briefly reviewing the dual optimization theorem and its associated corollaries, we used the equal magnitude property to extract the composition of a closed-form inverse, the "infinity inverse." The properties of this inverse, combined with a geometric knowledge of least infinity norm solutions, provided the foundations for a discussion of solution nonuniqueness and the potential for trajectory discontinuity as a result. We illustrated one possible method for "preemptive" avoidance of such discontinuities and attempted to provide examples of least infinity normbehavior throughout the text.

From this discussion and others in the current literature, it would seem that for any type of analysis involving polytope-like variable constraints, or any situation emphasizing the importance of a minimum maximal magnitude to an underdetermined system, the infinity norm is an invaluable tool. Almost every instance in the robotics literature where a pseudoinverse appears might represent a situation better served by a least infinity-norm solution, and subjects such as multiple-arm 
kinematics, optimal force distribution for cooperating manipulators, and multifingered grasping present but a small slice of the potential uses for least infinity norms. In point of fact, the work in [19] represents the case where a modified least infinity norm solution optimizes for minimum dynamic manipulator torques where previous attempts with pseudoinverses failed due to numerical instability [8]. It would seem that the infinity norm as an analysis tool may have more to offer than meets the eye.

\section{REFERENCES}

[1] I. Gravagne and I. D. Walker, "Properties of minimum infinity-norm optimization applied to kinematically redundant robots," in Proc. IEEE/RSJ Int. Conf. Intelligent Robots and Systems (IROS), Victoria, BC, Canada, Oct. 1998, pp. 152-160.

[2] — "Avoiding discontinuities while using the minimum infinity norm to resolve kinematic redundancy," in Proc. IEEE/ASME Int. Conf. Advanced Intelligent Mechatronics (AIM), Atlanta, GA, 1999.

[3] I. Gravagne, "Minimum effort techniques for inverse kinematics for redundant robot manipulators," M.S. thesis, Clemson Univ., Clemson, SC, May 1999.

[4] B. Siciliano, "Kinematic control of redundant robot manipulators: A tutorial," J. Intell. Robot. Syst., no. 3, pp. 201-212, 1990.

[5] D. N. Nenchev, "Redundancy resolution through local optimization: A review," J. Robot. Syst., vol. 6, no. 6, pp. 769-798, 1989.

[6] K. Doty, C. Melchiorri, and C. Bonivento, "A theory of generalized inverses applied to robotics," Int. J. Robot. Res., vol. 12, no. 1, pp. 1-19, Feb. 1993.

[7] T. Yoshikawa, "Analysis and control of robot manipulators with redundancy," in First International Symposium on Robotics Research. Cambridge, MA: MIT Press, 1984, pp. 735-747.

[8] J. M. Hollerbach and K. C. Suh, "Redundancy resolution of manipulators through torque optimization," IEEE J. Robot. Automat., vol. RA-3, pp. 308-316, Aug. 1987.

[9] B. J. Martin and J. E. Bobrow, "Determination of minimum-effort motions for general open chains," in Proc. IEEE Conf. Robotics and Automation, Nagoya, Japan, 1995, pp. 1160-1165.

[10] R. Finotello, T. Grasso, G. Rossi, and A. Terribile, "Computation of kinetostatic performances of robot manipulators with polytopes," in Proc. IEEE Conf. Robotics and Automation, Leuven, Belgium, 1998, pp. 3241-3246.

[11] V. C. Klema and A. J. Laub, "The singular value decomposition: Its computation and applications," IEEE Trans. Automat. Contr., vol. AC-22, no. 1, pp. 164-176, 1980

[12] A. S. Deo and I. D. Walker, "Minimum effort inverse kinematics for redundant manipulators," IEEE Trans. Robot. Automat., vol. 13, no. 5, pp. 767-775, 1997.

[13] — "Methods of redundancy resolution by infinity-norm minimization," in Proc. ISRAM'94. New York: ASME Press, 1994, pp. 67-74.

[14] J. A. Cadzow, "A finite algorithm for the minimum $\ell_{\infty}$-norm solution to a system of consistent linear equations," SIAM J. Numer. Anal., vol. 10, no. 4, pp. 607-617, 1973.

[15] — , "An efficient algorithmic procedure for obtaining a minimum $\ell_{\infty}$-norm solution to a system of consistent linear equations," SIAM J. Numer. Anal., vol. 11, no. 6, pp. 1151-1165, 1974.

[16] — " "Algorithm for the minimum-effort problem," IEEE Trans. Automat. Contr, vol. AC-16, pp. 60-63, Feb. 1971.

[17] — "Minimum-amplitude control of linear discrete systems," Int. J. Contr., vol. 19, no. 4, pp. 765-780, 1974.

[18] L. Kolev, "An algorithm of finite number of iterations for the minimum fuel and minimum amplitude control problems," Int. J. Contr., vol. 22, no. 1, pp. 97-102, 1975.

[19] I. C. Shim and Y. S. Yoon, "Stabilization constraint for torque optimization of a redundant manipulator," in Proc. IEEE Conf. Robotics and Automation (ICRA), Albuquerque, NM, Apr. 1997, pp. 2403-2408.

[20] C. A. Klein and C. H. Huang, "Review of pseudoinverse control for use with kinematically redundant manipulators," IEEE Trans. Syst., Man, Cybern., vol. SMC-13, pp. 245-250, Apr. 1983.

[21] H. Ding and S. K. Tso, "Minimum infinity-norm kinematic solution for redundant robots using neural networks," in Proc. IEEE Conf. Robotics and Automation, Leuven, Belgium, 1998, pp. 1719-1724.

[22] F. Chatelin, Eigenvalues of Matrices. New York: Wiley, 1993, pp. $44-45$.
[23] E. Kreyszig, Introductory Functional Analysis with Applications. New York: Wiley, 1989.

[24] A. Ben-Israel and T. Greville, Generalized Inverses: Theory and Applications. New York: Wiley, 1974.

[25] L. Cooper and D. Steinberg, Methods of Optimization. Philadelphia, PA: Saunders, 1970.

[26] G. Golub and C. F. Van Loan, Matrix Computations, 3rd ed. Baltimore, MD: John Hopkins Univ. Press, 1996.

[27] D. G. Luenberger, Optimization by Vector Space Methods. New York: Wiley, 1969.

[28] M. W. Spong and M. Vidyasagar, Robot Dynamics and Control. New York: Wiley, 1989.

\section{Iterative Regulation of an Electrically Driven Flexible-Joint Robot with Model Uncertainty}

\author{
Amit Ailon, Member, IEEE, Rogelio Lozano, Member, IEEE, and \\ Michael I. Gil'
}

Abstract-This study considers the set-point regulation control problem of rigid and flexible-joint electrically driven robots with model uncertainties and unknown payloads. The proposed control scheme is based on simple linear state and output feedbacks. The resulting controller can also be implemented in a case where the current signal is not measurable. Applications of the approach when the Coulomb friction forces are taken into account have also been considered.

Index Terms-Contraction mapping, flexible-joint robot, friction force, motor dynamics, output controller, set-point regulation, uncertainty.

\section{INTRODUCTION}

This study considers the set-point regulation problem of rigid-link electrically driven (RLED) and rigid-link flexible-joint electrically driven (RLFJED) robots. As shown in previous papers [14], [11], [7], [5], [6], the introduction of an electrical system between the control input and the torque actually applied to the link complicates the controller design in robotics. We assume here brushed DC (BDC) motors with known electrical circuit parameters [10, Sec. 6.4]. However the resulting control scheme overcomes the problem arising from the uncertainty of the mechanical model with an unknown payload.

The proposed control scheme for the set-point regulation problem is based on contraction mapping theory, which appears to be a useful framework for studying control problems in robotics. Applications of the contraction mapping method to robot control were first explored and demonstrated by [8], and later used in [1]. This paper further

Manuscript received July 28, 1999; revised March 15, 2000 and September 12, 2000. This paper was recommended for publication by Associate Editor F. C. Park and Editor A. De Luca upon evaluation of the reviewers' comments. This work was supported by the Paul Ivanir Center for Robotics Research and Production Management, Ben Gurion University of the Negev, Beer Sheva, Israel. The work of M. I. Gil was supported by the Israel Ministry of Science and Technology Kamea Fellowship. This paper was presented in part at the IEEE Conference on Control Applications, Trieste, Italy, September 1998.

A. Ailon is with the Department of Electrical and Computer Engineering, Ben Gurion University of the Negev, Beer Sheva 84105, Israel (e-mail: amit@ee.bgu.ac.il).

R. Lozano is with the Université de Technologie de Compiègne, 60206 Compiègne cédex, France.

M. I. Gil' is with the Department of Mathematics, Ben Gurion University of the Negev, Beer Sheva 84105, Israel.

Publisher Item Identifier S 1042-296X(00)11580-0. 